\title{
Implementation Performance of Tuberculosis Control in China: 2011-2020
}

\author{
Tao $\mathrm{Li}^{1, \alpha,} ; \mathrm{Xin} \mathrm{Du}^{1, \mathrm{~s}} ;$ Xiaoqiu Liu ${ }^{1}$; Yuhong $\mathrm{Li}^{1}$; Yanlin $\mathrm{Zhao}^{1, *}$
}

\section{Summary \\ What is already known about this topic? \\ World Health Organization (WHO) launched END \\ TB Strategy but performance of tuberculosis (TB) control in China hasn't been systematically evaluated after 2010. \\ What is added by this report? \\ All five key indicators monitorred in national TB program (NTP) have kept with high level or got impressively improved from 2011 to 2020. There were some differences in the performance of indicators among different regions. \\ What are the implications for public health practice? \\ NTP indicators should be readapted to new strategies and requirements in future plan.}

The results from previous national surveys showed that China had made great progress in $\mathrm{TB}$ control from 1990 to 2010 and had achieved the United Nations (UN) Millennium Development Goals (MDGs) targets before the target date (halving prevalence and mortality rates by 2015 compared with 1990 levels) (1). A new TB service network integrating "prevention, treatment, management, and patient care" has been established after that. In 2015, the World Health Organization (WHO) launched the post-2015 era END TB Strategy, in which 2020, 2035, and 2050 incidence and mortality milestones have been settled to measure global and regional progress in comparison to 2015 levels (2). There is a requirement for the national TB program (NTP) to evaluate its stage progress. Key indicators were derived from notification data collected from the national Tuberculosis Information Management System (TBIMS) (3). All five key indicators have maintained high levels or were significantly improved in the past decade. There were still some differences in the performance of indicators among different regions. The 14th Five-Year Plan will be a new start and NTP indicators should be readapted to the new strategies and requirements. The implications and definitions of the overall arrival rate should be changed. More efficient diagnostic tests should be scaled up to increase the bacteriologicallypositive rate. Furthermore, the current two separated drug resistance screening indicators could be combined together.

Analytical notification data was extracted from TBIMS, and 5 key indicators were calculated based on cases between January 1, 2011 and December 31, 2020. Considering the socioeconomic circumstances varying among the 31 provincial-level administrations (PLADs), the PLADs were divided into 4 regions based on general social development indicators: JJH (Beijing, Tianjin, and Shanghai); East (Hebei, Liaoning, Jiangsu, Zhejiang, Fujian, Shandong, Guangdong, and Hainan); Central (Shanxi, Jilin, Heilongjiang, Anhui, Jiangxi, Henan, Hubei, and Hunan); West (Inner Mongolia, Guangxi, Chongqing, Sichuan, Guizhou, Yunnan, Tibet (Xizang), Shaanxi, Gansu, Qinghai, Ningxia, and Xinjiang).

The 5 key indicators were as follows:

1) Overall arrival rate was defined as the proportion of cases that ultimately arrived at TB designated health facilities among all pulmonary tuberculosis (PTB) or presumptive PTB cases reported in the National Notifiable Disease Reporting System (NNDRS) by non-designated health facilities. 2) Bacteriologicallypositive rate was defined as the proportion of bacteriologically-confirmed cases through sputum smear, culture, or molecular tests among all PTB patients notified in TBIMS, with TB pleurisy not included. 3) PTB treatment success rate referred to the proportion of patients with "cured" or "treatment completed" outcomes after finishing standard treatment course among all PTB patients notified in TBIMS. 4) Drug susceptibility test (DST) -multidrug resistant (MDR)-TB high-risk population referred to the proportion of cases tested for rifampicin resistance among all MDR-TB high-risk populations notified in TBIMS. 5) DST-new cases was defined as the proportion of cases tested for rifampicin resistance among all new bacteriologically confirmed cases 
notified in TBIMS.

The overall arrival rate increased from $93.5 \%$ in 2011 to $97.3 \%$ in 2020 nationwide (Table 1). The bacteriologically-positive rate initially declined then rebounded in past decades to finally reach $55.3 \%$ in 2020 (Figure 1). The treatment success rate of more than 7 million PTB patients involved in NTP from 2011 to 2019 was continuously higher than $93 \%$ (Figure 2). DST-MDR-TB high-risk populations and DST-new cases both started from low levels to reach levels higher than $90 \%$ (Table 1).

The performance of indicators in different regions did not always show the same trend nationwide except for overall arrival rate. The bacteriologically-positive rate in the $\mathrm{JJH}$ region has changed from the lowest (39.4\%) in 2011 to the highest $(58.6 \%)$ in comparison to that of other regions while its treatment successful rates were almost always the lowest in the whole period. JJH showed good performance in DST indicators for both high-risk populations and new bacteriologically-confirmed cases from the beginning even they also varied during the middle sections of the study period. On the contrary, the starting level of
DST indicators in East, Central, and West regions was relatively low; however, they quickly increased to more than $90 \%$ after descending in the first few years. Generally, indicators in the Central and East regions were higher than those in the West region, except that West PLADs showed better performance than central PLADs in the indicator of "DST-new cases".

\section{DISCUSSION}

TB control in China continues making great progress since 2011. Five key indicators have maintained high levels or improved significantly. Based on these performances, the target of decreasing the incidence rate of PTB that was marked in the "13th Five-Year Plan for National TB Control (2016-2020)" has already been successfully completed, and its annual avarege decline rate $(3.2 \%)$ is significantly higher than the global average (1.7\%) (4).

The proportion of reported PTB and presumptive PTB cases arriving at designated hospitals maintained a high level after 2011. The high performance of referral work is the premise of guaranteeing patients involved

TABLE 1. The change of overall arrival rate and DST proportions in China and sub-national regions, 2011-2020 (\%)

\begin{tabular}{|c|c|c|c|c|c|c|c|c|c|c|}
\hline Item & 2011 & 2012 & 2013 & 2014 & 2015 & 2016 & 2017 & 2018 & 2019 & 2020 \\
\hline \multicolumn{11}{|l|}{ Overall arrival rate, $\%$} \\
\hline Nationwide & 93.5 & 94.7 & 95.1 & 89.5 & 90.2 & 90.9 & 90.7 & 93.4 & 96.0 & 97.7 \\
\hline JJH & 92.6 & 92.9 & 92.6 & 87.4 & 91.7 & 86.3 & 93.3 & 94.4 & 95.2 & 97.0 \\
\hline East & 92.2 & 93.8 & 94.9 & 91.1 & 91.6 & 91.3 & 90.3 & 91.9 & 94.7 & 97.6 \\
\hline Central & 96.6 & 96.8 & 97.7 & 92.8 & 93.1 & 94.7 & 94.0 & 94.9 & 96.8 & 97.7 \\
\hline West & 92.6 & 94.3 & 94.7 & 85.9 & 86.7 & 88.3 & 88.5 & 93.4 & 96.6 & 97.8 \\
\hline \multicolumn{11}{|c|}{ DST-MDR-TB high-risk populations, $\%$} \\
\hline Nationwide & - & - & - & 35.7 & 73.2 & 54.8 & 57.2 & 72.6 & 88.4 & 97.4 \\
\hline JJH & - & - & - & 91.2 & 88.9 & 70.8 & 69.1 & 84.3 & 91.8 & 97.0 \\
\hline East & - & - & - & 49.1 & 75.7 & 62.0 & 67.2 & 81.4 & 90.7 & 97.4 \\
\hline Central & - & - & - & 36.2 & 76.2 & 50.6 & 57.4 & 69.5 & 84.1 & 97.3 \\
\hline West & - & - & - & 16.0 & 66.3 & 48.2 & 45.2 & 65.7 & 89.3 & 97.5 \\
\hline \multicolumn{11}{|l|}{ DST-new cases, $\%$} \\
\hline Nationwide & - & - & - & 18.8 & 26.7 & 22.8 & 33.1 & 60.2 & 80.4 & 93.0 \\
\hline JJH & - & - & - & 57.5 & 28.5 & 45.5 & 48.0 & 85.6 & 95.4 & 95.4 \\
\hline East & - & - & - & 24.9 & 26.4 & 28.6 & 45.6 & 72.9 & 84.3 & 93.4 \\
\hline Central & - & - & - & 14.4 & 22.8 & 17.7 & 23.4 & 50.1 & 74.2 & 91.2 \\
\hline West & - & - & - & 16.2 & 32.9 & 21.1 & 31.0 & 55.6 & 81.5 & 94.1 \\
\hline
\end{tabular}

Note: JJH: Beijing, Tianjin, and Shanghai; East: Hebei, Liaoning, Jiangsu, Zhejiang, Fujian, Shandong, Guangdong, and Hainan; West: Inner Mongolia, Guangxi, Chongqing, Sichuan, Guizhou, Yunnan, Tibet (Xizang), Shaanxi, Gansu, Qinghai, Ningxia, and Xinjiang, Ningxia, and Xinjiang; -: not available.

Abbreviations: DST=drug susceptibility test; PTB=pulmonary tuberculosis; MDR-TB=multidrug resistant tuberculosis.

* Monitoring of DST proportion of MDR-TB started from 2014. 


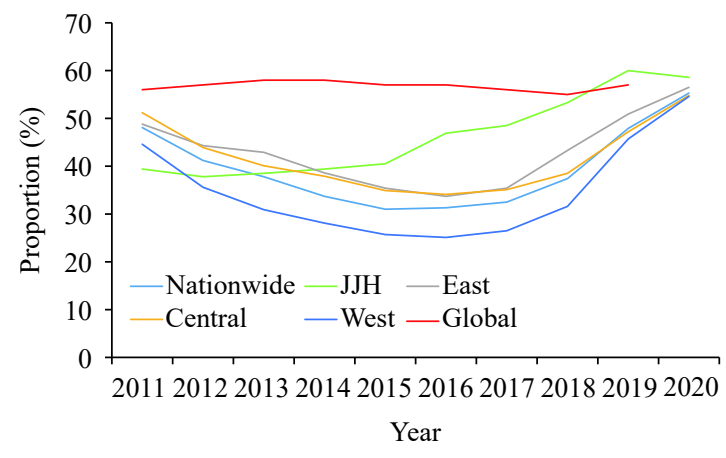

FIGURE 1. The change of bacteriologically-positive rate in China and sub-national regions comparing to global level, 2011-2020.

Note: JJH: Beijing, Tianjin, and Shanghai; East: Hebei, Liaoning, Jiangsu, Zhejiang, Fujian, Shandong, Guangdong, and Hainan; Central: Shanxi, Jilin, Heilongjiang, Anhui, Jiangxi, Henan, Hubei, and Hunan; West: Inner Mongolia, Guangxi, Chongqing, Sichuan, Guizhou, Yunnan, Tibet (Xizang), Shaanxi, Gansu, Qinghai, Ningxia, and Xinjiang.

in NTP diagnosis cascade and initiation of standardized treatment. Meanwhile, the adaption of this indicator also reflected essential implication for China's NTP patients management work. Before 2014, the overall arrival rate was only monitored among TB patients reported in resident county. With expansion to reported patients outside resident counties, NTP has greatly enhanced its migrant patients management since 2014. Therefore, it declined at the beginning of strategy changing but quickly rebounded and reached at $97.7 \%$ at the end of 2020 .

The proportion of bacteriologically-confirmed cases among PTB patients has raised to $55.3 \%$ in China in 2020. It has already met the requirement $(50 \%)$ posted in the National "Stop TB Action Plan (2019-2022)" (5) but was still relatively low comparing to other countries. In 2019, bacteriologically-confirmed cases accounted for $57 \%$ of PTB worldwide and about $80 \%$ in high-income countries (4). This indicator also fluctuated, which may be related with the changing of TB diagnosis and treatment service models. General hospitals historically preferred to use imaging methods to clinically diagnose $\mathrm{TB}$ rather than perform bacteriological examinations for every patient. Therefore, the bacteriologically-positive rate has declined more than one-third nationwide in the first serveral years. The decline was also affected by the scale of system reforming. In the West where most treatment centers were previous CDCs and shifted to general hospitals, the bacteriologically-positive rate declined by almost half. While in JJH areas where the service systems were relatively stable, the rate only declined slightly at the beginning and then increased step by step.

Another indicator with a stable high level is the treatment success rate that contributed to a relatively low TB death burden. The mortality rate in China fell by $35.3 \%$ from 2011 to 2019 and became the lowest in 30 High Burden Countries (4). But several challenges still exist. Ageing could be a serious problem in the future for China's TB control, especially in big cities and eastern PLADs (6). In our study, the relative lower treatment success rate in the East and JJH (Beijing, Shanghai and Tianjing) also remain concerning.

The coverage of drug susceptibility tests increased significantly in recent years. The proportion of drug resistance test screening among high-risk populations has reached a high level, which can be attributed to the continuously strengthened drug-resistant $\mathrm{TB}$ control strategies, e.g. scaling up the coverage of new diagnostic tools, involving screening expenditure to social medical insurances. As a result, the number of identified laboratory-confirmed rifampicin resistant (RR)-TB or MDR-TB cases has increased from 5,807 in 2014 to 18,246 in 2019 (4). But on the other hand, we should also notice that it is not enough to only screen among high-risk populations. The RR rate of new patients in China is also high and has raised from $6.7 \%$ in 2007 (7) to $7.1 \%$ in 2013 (8), which is about 2 times the average level of MDR-TB high burden countries. These types of patients are especially concerning and need to be found among new patients.

Our study has limitations. These five key indicators can not describe the whole complete frame of China's NTP practice. There were still many challenges and barriers that need to be carefully analyzed and addressed in future studies. In addition, our study only focuses on the performance of patients involved in the national surveillance system. There were still considerable TB patients not notified in TBIMS (9) that need to be further investigated.

All five key indicators has been greatly improved in the past decade, but the road towards a "Zero TB" world remains difficult. The 14 th five-year plan will be a new start and NTP indicators should be readapted to new strategies and requirements. More and more designated centers were referred to general hospitals, and institutional referral may not be the focus for patient detection. Instead, all the reported patients should be restrictly involved in NTP management and the implication and definition of the overall arrival rate 


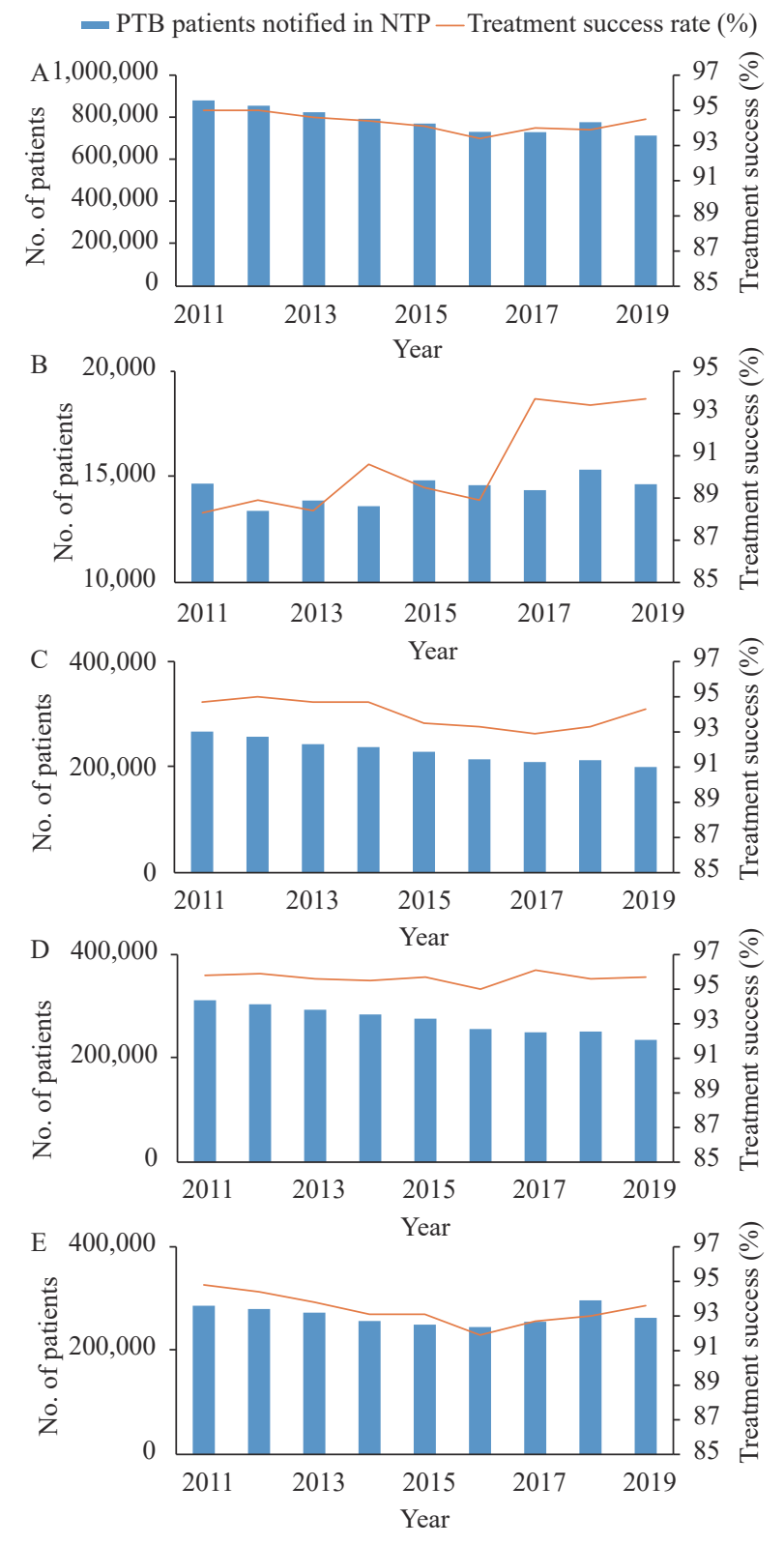

FIGURE 2. Number and treatment success rate of PTB patients notified in NTP in China and subnational areas, 2011-2019. (A) Nationwide; (B) JJH (Beijing, Shanghai and Tianjing); (C) East; (D) Central; (E) West.

Note: Result of successful treatment rate for PTB patients notified in 2020 cannot be evaluated yet at the moment of assessment. JJH: Beijing, Tianjin, and Shanghai; East: Hebei, Liaoning, Jiangsu, Zhejiang, Fujian, Shandong, Guangdong, and Hainan; Central: Shanxi, Jilin, Heilongjiang, Anhui, Jiangxi, Henan, Hubei, and Hunan; West: Inner Mongolia, Guangxi, Chongqing, Sichuan, Guizhou, Yunnan, Tibet, Shaanxi, Gansu, Qinghai, Ningxia, and Xinjiang. Abbreviations: PTB=pulmonary tuberculosis; NTP=national TB program.

should be changed accordingly. Monitoring and scaling up more efficient diagnostic tests (e.g. rapid molecular tests) to increase the bacteriologically- positive rate will ensure patients are correctly diagnosed and receive effective treatment as early as possible, which can both benefit treatment outcomes and be the premise of drug resistance screening. Furthermore, the two separated drug resistance screening indicators can be combined into DST proportion among bacteriologically-positive patients.

Acknowledgments: Participants of the national TB surveillance system.

Funding: The China National TB Program and the National Special Science and Technology Project for Major Infectious Diseases of China (2017ZX10201302007).

Conflicts of interest: All authors have completed and submitted the ICMJE form. And no conflicts of interest were reported.

doi: $10.46234 /$ ccdcw 2021.073

\# Corresponding author: Yanlin Zhao, zhaoyl@chinacdc.cn.

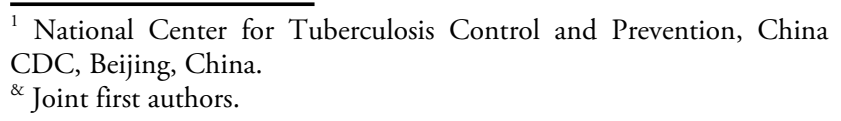

Submitted: February 19, 2021; Accepted: March 17, 2021

\section{REFERENCES}

1. World Health Organization. Global tuberculosis control: WHO report 2011. Geneva, Switzerland: WHO; 2011. WHO/HTM/TB/2011. 12011.16. https://apps.who.int/iris/bitstream/handle/10665/44728/978 9241564380_eng.pdf?sequence=1. [2021-03-16].

2. Uplekar M, Weil D, Lonnroth K, Jaramillo E, Lienhardt C, Dias HM, et al. WHO's new end TB strategy. Lancet 2015;385(9979):1799-801. https://www.sciencedirect.com/science/article/abs/pii/S01406736156057 00 .

3. Huang F, Cheng SM, Du X, Chen W, Scano F, Falzon D, et al. Electronic recording and reporting system for tuberculosis in China: experience and opportunities. J Am Med Inform Assoc 2014;21(5): 938-41. https://pubmed.ncbi.nlm.nih.gov/24326537/.

4. World Health Organization. Global tuberculosis report 2020. Geneva, Switzerland: WHO; 2020. https://apps.who.int/iris/handle/10665/ 336069. [2021-03-16].

5. National Health Commission. Stop TB Action Plan (2019-2022). http://www.nhc.gov.cn/jkj/s3589/201906/b30ae2842c5e4c9 ea2f9d5557ad4b95f.shtml. [2019-6-13]. (In Chinese).

6. Zhu WL, Wang YS, Li T, Chen W, Wang WB. Gap to End-TB targets in eastern China: a joinpoint analysis from population-based notification data in Zhejiang Province, China, 2005-2018. Int J Infect Dis 2021;104:407 - 14. http://dx.doi.org/10.1016/j.ijid.2021.01.007.

7. Zhao YL, Xu SF, Wang LX, Chin DP, Wang SF, Jiang GL, et al. National survey of drug-resistant tuberculosis in China. N Engl J Med 2012;366(23):2161-70. https://pubmed.ncbi.nlm.nih.gov/22670902/.

8. World Health Organization. Global tuberculosis report 2017. Geneva, Switzerland: WHO; 2017. WHO/HTM/TB/2017.23. https://relie fweb.int/sites/reliefweb.int/files/resources/9789241565516-eng.pdf.

9. Li T, Shewade HD, Soe KT, Rainey JJ, Zhang H, Du X, et al. Underreporting of diagnosed tuberculosis to the national surveillance system in China: an inventory study in nine counties in 2015. BMJ Open 2019;9(1):e021529. https://pubmed.ncbi.nlm.nih.gov/30696665/. 